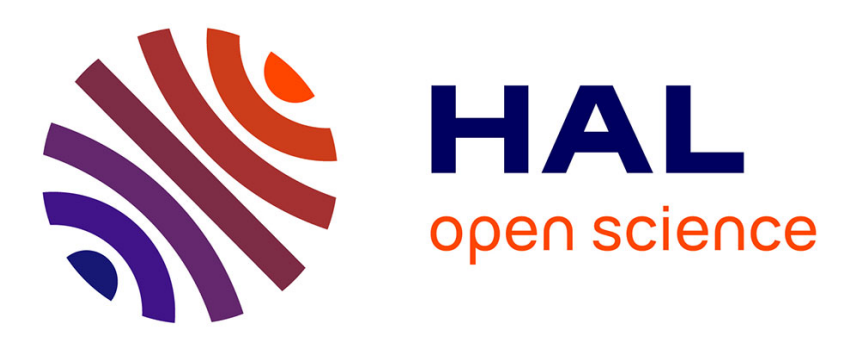

\title{
Ganin ya fi ji / Voir est mieux qu'entendre: lire l'identité sur la peau (Sahel central, XIXe siècle)
}

Camille Lefebvre

\section{To cite this version:}

Camille Lefebvre. Ganin ya fi ji / Voir est mieux qu'entendre: lire l'identité sur la peau (Sahel central, XIXe siècle) . Critique Internationale, 2015, Voir l'histoire: sources visuelles et écriture du regard, 3 (68), pp.39-59 10.3917/crii.068.0039 . halshs-01511057

\section{HAL Id: halshs-01511057 https://shs.hal.science/halshs-01511057}

Submitted on 20 Apr 2017

HAL is a multi-disciplinary open access archive for the deposit and dissemination of scientific research documents, whether they are published or not. The documents may come from teaching and research institutions in France or abroad, or from public or private research centers.
L'archive ouverte pluridisciplinaire HAL, est destinée au dépôt et à la diffusion de documents scientifiques de niveau recherche, publiés ou non, émanant des établissements d'enseignement et de recherche français ou étrangers, des laboratoires publics ou privés. 


\section{Critique \\ internationale \\ Revue comparative de sciences sociales}

\section{6}

\section{Voir l'histoire : sources visuelles et écriture du regard}

Dialogues avec le visible : I'historien et le regard par Nadège Ragaru

Les atlas historiques de ville et l'administration du passé métropolitain au XIX' siècle par Stéphane Van Damme

Ganin ya fi ji / Voir est mieux qu'entendre : lire l'identité sur la peau (Sahel central, XIX' siècle) par Camille Lefebvre

La voix et le regard : les régimes visuels des concours d'autobiographies polonais, 1930-1984 par Katherine Lebow

Voir et devoir voir le passé. Retour sur une exposition historique à visée commémorative par Sarah Gensburger 
Numéro 68 - juillet-septembre 2015

Trimestriel

\section{Critique \\ internationale}

Revue comparative de sciences sociales 
Critique internationale

Revue comparative de sciences sociales

\section{Rédaction}

CERI. 56 rue Jacob, 75006 Paris

Tél. 01587170 77. Fax. 0158717091

catherine.burucoa@sciencespo.fr

sciencespo.fr/ceri/fr/critique

Rédactrice en chef Laurence Louër

Responsable de la rubrique Lectures Nadège Ragaru Responsable éditoriale Catherine Burucoa

Rédaction Laetitia Atlani-Duault, Antonela CapellePogăcean, Hélène Combes, Nathalie Duclos, Gilles Favarel-Garrigues, François Foret, Chloé Froissart, Laurent Gayer, †Bastien Irondelle, Jeanne Lazarus, Sébastien Lechevalier, Benjamin Lemoine, Catherine Perron, Sandrine Perrot, Franck Petiteville, Nadège Ragaru, Sandrine Revet, Antoine Roger, Hélène Thiollet, Antoine Vauchez

\section{Conseil scientifique}

Président Frédéric Mion

Alban Bensa, John R. Bowen, Hamit Bozarslan, JeanLuc Domenach, A.J.R. Groom, Gérard Grunberg, Pierre Hassner, Christopher Hill, Christophe Jaffrelot, Sunil Khilnani, Jean Leca, David Lehmann, Thomas Lindenberger, Andreas Mehler, Anand Menon, JeanLuc Parodi, Pablo A. Piccato, Jennifer Pitts, Vivien Schmidt, Roger de Weck

Directeur de la publication Alain Dieckhoff

\section{Édition, ventes et abonnements}

Presses de Sciences Po

117 boulevard Saint-Germain, 75006 Paris

Tél. 01454983 64. Fax. 0145498334

info.presses@sciences-po.fr

Tarifs d'abonnement et bon de commande p. 201

(c) Presses de Sciences Po

Maquette Conception Ghislaine Garcin

Couverture et illustrations Elsa Mathern

Critique internationale est une revue de Sciences Po (Fondation nationale des sciences politiques et Institut d'études politiques de Paris), publiée avec le concours du Centre national de la recherche scientifique et du Centre national du livre.

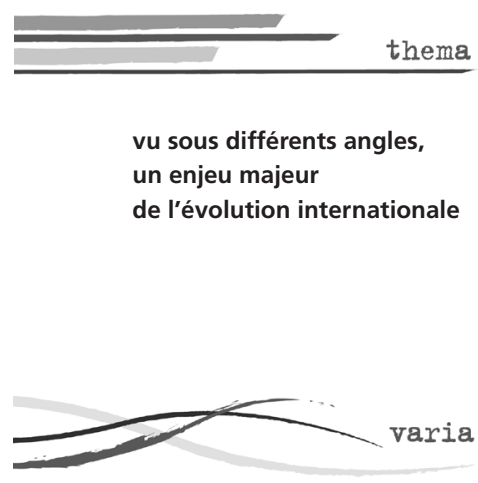

des essais, des enquêtes, l'état de la recherche, des documents originaux, des entretiens

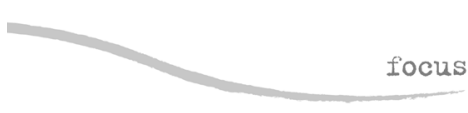

des événements de l'actualité internationale remis en perspective

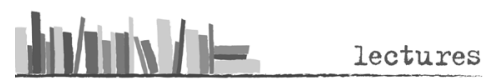

un aperçu de la recherche en mouvement 


\section{Ganin ya fi ji / Voir est mieux qu'entendre : lire l'identité sur la peau (Sahel central, $\mathrm{XIX}$ siècle)}

par Camille Lefebvre

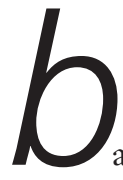

alafres ethniques, scarifications tribales, tatouages de race, cicatrices raciales, en anglais, le plus souvent, tribal marks, les désignations coloniales des pratiques de scarifications se réduisent souvent à une lecture univoque. Malgré leur diversité dans le temps et dans l'espace, ces pratiques apparaissent dans les discours coloniaux comme des usages figés et immuables par lesquels on aurait toujours, et de la même manière, figuré l'ethnie sur la peau. Néanmoins, dès le départ, les observateurs coloniaux se heurtent à l'impossibilité de mettre leur théorie en pratique et constatent que ces marques ont perdu « une grande partie de leur signification ethnique », probablement, selon eux, en raison de l'enchevêtrement des races et de la paix instaurée par la colonisation ${ }^{1}$. Ainsi, loin d'amener à remettre en cause le biais ethnique, le fait de ne pouvoir lire mécaniquement l'ethnie sur la peau est expliqué par une perte des traditions qui aurait fait oublier aux porteurs eux-mêmes le sens originel de tels signes. Stigmatisée dès lors comme un outil inutile à l'ethnologie ou à l'anthropologie, cette pratique est constamment décrite comme étant vouée à disparaître sous l'effet de la modernité coloniale.

1. Docteur Jules Gaston Decorse, «Le tatouage : les mutilations ethniques et la parure chez les populations du Soudan », L'antbropologie, 16, 1905, p. 134-135; Docteur L. Pales, « Les mutilations tégumentaires en Afrique noire », Fournal de la Société des Africanistes, 16, 1946, p. 3. 
Au-delà d'une analyse des discours coloniaux sur les scarifications, le renouveau récent des études historiques sur le tatouage dans les mondes occidentaux et dans le Pacifique invite à porter un autre regard sur ces pratiques de modifications corporelles. En effet, ces travaux ont montré la valeur heuristique non seulement d'une analyse de leur nature changeante dans le temps mais aussi de la prise en compte des différentes significations du tatouage en fonction des sociétés dans lesquelles il est mis en œuvre ${ }^{2}$. Au sein des études africaines, exception faite de l'histoire de l'art et de quelques travaux en anthropologie, cet objet a longtemps été marqué, selon la formule de Megan Vaughan, par une forme d'« anxiété académique $»^{3}$. Au tournant des années 2000, Heidi Gengenbach a fait des pratiques de scarification des objets d'histoire en montrant que leur analyse permettait d'appréhender l'expérience intime des femmes pendant la colonisation portugaise ${ }^{4}$. À sa suite, M. Vaughan a proposé d'étudier ces pratiques pour réfléchir aux multiples façons dont les sociétés précoloniales marquaient la différence et l'identité individuelle et sociale . Dans l'espace qui nous intéresse, le Sahel central, les travaux pionniers de Paul Lovejoy ont, dès la fin des années 1970, révélé comment les scarifications étaient utilisées au milieu du XIX ${ }^{\mathrm{e}}$ siècle par certaines communautés dans le jeu complexe des identités. En effet, les groupes migrants Agalawa, Tokarawa et Kambarin Beriberi, pratiquant le commerce de la cola et récemment intégrés au sein des sociétés haoussas, mobilisaient différentes identités selon les situations. Ils s'affirmaient tantôt comme musulmans, tantôt comme Haoussas ou d'origine bornouane ou touarègue et conservaient pour cela les scarifications liées à ces multiples identités ${ }^{6}$. Récemment, plusieurs chercheurs travaillant sur la traite atlantique ont proposé d'utiliser les traces des scarifications, conservées notamment dans des registres, comme des moyens de retrouver des histoires individuelles dans le cadre d'un système qui faisaient disparaître les individus ${ }^{7}$. Cependant, si ces

2. Alfred Gell, Wrapping in Images: Tattooing in Polynesia, Oxford, Clarendon Press, 1993 ; Jane Caplan (ed.), Written on the Body: The Tattoo in European and American History, Princeton, Princeton University Press, 2000.

3. Paul Bohannan, «Beauty and Scarification amongst the Tiv», Man, 56, 1956, p. 117-121; Thierry Maertens, Le dessein sur la peau : essai d'antbropologie des inscriptions tégumentaires, Paris, Aubier-Montaigne, 1978 ; Arnold Rubin (ed.), Marks of Civilization: Artistic Transformations of the Human Body, Los Angeles, Museum of Cultural History/University of California, 1988 ; Michèle Coquet, « L'envers du regard », Fournal des africanistes, 64 (2), 1994, p. 39-63 ; Joseph Nevadomsky, Ekhaguosa Aisien, « The Clothing of Political Identity: Costume and Scarification in the Benin Kingdom », African Arts, 28 (1), 1995, p. 62-73 ; Olanikẹ Ola Orie, « The Structure and Function of Yoruba Facial Scarification », Anthropological Linguistics, 53 (1), 2011, p. 15-33.

4. Heidi Gengenbach, « Boundaries of Beauty: Tattooed Secrets of Women's History in Magude District, Southern Mozambique », Fournal of Women's History, 14 (4), 2003, p. 106-141.

5. Megan Vaughan, «Scarification in Africa. Re-reading Colonial Evidence », Cultural and Social History. The Fournal of the Social History Society, 4 (3), 2007, p. 390.

6. Paul Lovejoy, Caravans of Kola: The Hausa Kola Trade, 1700-1900, Zaria, Ahmadu Bello University Press, 1980, p. 84-85 ; P. Lovejoy, « The Kambarin Beriberi: The Formation of a Specialized Group of Hausa Kola Traders in the Nineteenth Century », The Journal of African History, 14 (4), 1973, p. 636.

7. Katrina H. B. Keefer, « Scarification and Identity in the Liberated Africans Department Register, 1814$1815 »$, Canadian Fournal of African Studies, 47 (3), 2013, p. 538 ; P. Lovejoy, « Scarification and the Loss of History in the African Diaspora », dans Andrew Apter, Lauren Derry (eds), Activating the Past Historical Memory in the Black Atlantic, Newcastle, Cambridge Scholarly Publishing, 2009, p. 99-138 ; Henry Lovejoy, «The Registers of Liberated Africans of the Havana Slave Trade Commission: Transcription Methodology and Statistical Analysis », African Economic History, 38 (1), 2010, p. 107-135 ; H. Lovejoy, « Old Oyo Influences on the Transformation of Lucumí Identity in Colonial Cuba », Ph. D. University of California, 2012. 
scarifications sont le reflet d'un langage complexe de l'identité qui était dans une large mesure lisible et accessible aux contemporains informés, faire une lecture positive de ces marques comporte le risque de les imaginer comme des traces qu'il nous suffirait de déchiffrer pour accéder à un savoir enfoui. Or ces traces nous échappent en partie, certaines lacunes de la documentation ne peuvent être résolues et nous ne pouvons les comprendre sans questionner les usages et les pratiques sociales complexes dont elles sont le reflet.

Pour cela, il est nécessaire de renouveler notre documentation et d'attirer dans le champ de notre narration des documents inattendus. En effet, les séries de gravures ou de dessins des voyageurs du XIX ${ }^{\mathrm{e}}$ siècle, les collections de dessins anthropologiques et anthropométriques ou les descriptions ethnographiques de la période coloniale n'offrent souvent que des données descriptives, fragmentaires et décontextualisées qui se dérobent à l'interprétation ${ }^{8}$. Les enquêtes orales ne comblent pas totalement ces manques et ne permettent pas non plus d'accéder à la question du sens de ces pratiques dans le passé . Pour dépasser ces dessins schématiques, l'accumulation comptable du nombre de marques selon les lieux et les groupes et une interprétation de l'ordre du lieu commun qui fait de ces marques un outil de reconnaissance, un détour par les usages vernaculaires et les manières dont on parlait alors de cette pratique est indispensable. Pour accéder à ces usages, l'historien du Sahel au $\mathrm{XIX}^{\mathrm{e}}$ siècle dispose d'une série de matériaux en haoussa et en kanouri recueillis par des savants amateurs et des linguistes entre 1772 et 1918. En effet, parallèlement à la curiosité pour la géographie de l'intérieur du continent africain, qui motive les projets d'exploration depuis la seconde moitié du XVIII ${ }^{\mathrm{e}}$ siècle, se développe un intérêt pour les langues africaines, leur identification, leur recueil et leur description. Durant tout le $\mathrm{XIX}^{\mathrm{e}}$ siècle et au début du $\mathrm{XX}^{\mathrm{e}}$, la volonté de décrire la grammaire à partir d'éléments conséquents de la langue pousse à recueillir la parole libre de locuteurs afin de bénéficier d'exemples en contexte. Les paroles gelées dans ces sources permettent un regard inédit sur les scarifications faciales dans le Sahel central du $\mathrm{XIX}^{\mathrm{e}}$ siècle et les révèlent comme pratiques sociales et politiques ancrées dans un temps et un lieu donnés.

\section{Lire des dessins}

Pour les explorateurs, britanniques et germanophones, qui parcourent la région entre 1798 et 1890 , la question des scarifications faciales est un sujet marginal au regard de leurs préoccupations marquées pour les sujets géographiques, politiques

8. Parmi les dessins figurant des visages et ou des corps scarifiés, seuls ont été utilisés ici ceux pour lesquels nous disposons du nom de l'individu et/ou d'une indication d'origine géographique précise. 129 dessins sont concernés pour lesquels, dans 116 cas, l'individu représenté est nommé et pour tous est indiqué un lieu de naissance ou de résidence.

9. J'ai mené une enquête sur ces questions à Niamey (Niger) en septembre 2013. Les résultats de cette enquête étaient très riches sur les usages récents de ces pratiques mais n'offraient que peu de données concluantes sur le XIX ${ }^{\mathrm{e}}$ siècle. 
et économiques. C'est souvent à l'occasion d'une rencontre ou lorsqu'ils remarquent un fait qui sort de l'ordinaire qu'ils évoquent, en passant, ces pratiques de modifications corporelles. Pour l'explorateur George Francis Lyon, beaucoup d'Arabes du désert (Libye actuelle) auraient le visage couvert de cicatrices liées à des brulures thérapeutiques ou amoureuses ${ }^{10}$. En 1822, Hugh Clapperton qualifie la pratique du tatouage de commune chez toutes les nations noires ${ }^{11}$. Trente ans plus tard, James Richardson observe que «se scarifier le visage ou le cou prévaut partout au Bornou, au Soudan et dans toute cette partie de l'Afrique, les Touaregs et les Fellatahs étant les seules populations à s'abstenir de cette pratique barbare $\gg^{12}$. En 1885-1886, Paul Staudinger remarque que beaucoup d'hommes et de femmes haoussas portent des tatouages décoratifs ${ }^{13}$. Au-delà de ces considérations générales, tous soulignent la présence de populations ou d'individus qui, contrairement aux autres, ne se scarifient pas. Richardson note par exemple que la femme du chef de la ville de Ghât n'a pas le visage défiguré par les tatouages si commun à toutes les beautés sahariennes, et Heinrich Barth évoque des Budumas aux beaux traits intacts de toute incision ${ }^{14}$.

Ces indications récurrentes de populations ne pratiquant pas de marquages varient selon les observateurs. Ainsi, pour Barth, en 1851, les Budumas ne portent pas de marques, mais pour Gustav Nachtigal, qui voyage vingt ans après, ils ont deux incisions au coin des yeux, et pour les anthropologues de la mission Tilho en 1907, « les tatouages de race sont [chez eux] presque constants » : deux traits à la queue du sourcil, deux autres au-dessus des pommettes et deux en dessous se rapprochant des sillons naso-géniens ${ }^{15}$. Pour Richardson, les noirs de Kano ne se scarifient pas le visage, mais il est le seul à évoquer cette spécificité, de nombreux autres observateurs dessinant ou évoquant les marques portées par les Kanawa $^{16}$. Pour Lyon, ce sont les Toubous qui ne se scarifient pas, mais Dixon Denham décrit précisément des motifs aux connotations statutaires portés par les

10. George Francis Lyon, A Narrative of Travels in Northern Africa in the Years 1818-1819 and 1820, Londres, John Murray, 1821, p. 51-52.

11. Hugh Clapperton, dans Dixon Denham, Hugh Clapperton, Walter Oudney, Discoveries in Northern and Central Africa in Years 1822, 1823, and 1824, Londres, John Murray, 1826, t. 1, p. 144.

12. James Richardson, Narrative of a Mission to Central Africa Performed in the Years 1850-1851, Londres, Chapman and Hall, 1853, t. 2, p. 96.

13. Paul Staudinger, In the Heart of the Hausa States, Athens, Ohio University Center for International Studies, 1990 (1889), t. 2, p. 209.

14. J. Richardson, Travels in the Great Desert of Sahara, in the Years of 1845 and 1846, Londres, R. Bentley, 1848, t. 2, p. 413 ; Heinrich Barth, Travels and Discoveries in North and Central Africa. Being a Fournal of an Expedition Undertaken under the Auspices of H.B.M.'s Government, in the Years 1849-1855, Londres, Franck Cass, 1965 (facsimilé du centenaire de l'édition de 1865), t. 2, p. 53.

15. Allan G. B. Fisher, Humphrey J. Fisher, Sahara and Sudan. Volume III. The Chad Basin and Bagirmi by Gustav Nachtigal, Londres, Hurst \& Company, 1987, p. 112 ; Raoul Gaillard, Léon Poutrin, «Étude anthropologique des populations des régions du Tchad et du Kanem », dans Capitaine Tilho, Documents scientifiques de la Mission Tilho (1906-1909), Paris, Imprimerie nationale, 1909, t. 3, p. 21.

16. J. Richardson, Narrative of a Mission to Central Africa Performed in the Years 1850-1851, op. cit., t. 1, p. 222. 
Toubous Gundas ${ }^{17}$. Indépendamment des possibilités d'erreurs d'observation, cette diversité des regards témoigne du fait que les pratiques de scarifications évoluent régulièrement. Certains groupes ou certaines familles peuvent en abandonner l'usage pour un temps. Une mère qui aura perdu plusieurs enfants portant une marque particulière cessera de la faire tracer sur le visage des enfants suivants et pourra en adopter une autre ${ }^{18}$. Il arrive également qu'un groupe entier, comme les Kouris au début du $\mathrm{XX}^{\mathrm{e}}$ siècle, s'abstienne à un moment donné de se scarifier parce qu'il considère, en raison de circonstances particulières, que cela porte malheur ${ }^{19}$. Si les observateurs ne s'accordent pas sur les groupes ou les individus qui ne portent pas de marques, leurs descriptions ne concordent pas non plus sur les marques qui sont portées. On constate régulièrement des différences entre les descriptions ou les dessins, soit dans le nombre, soit dans la configuration formelle des cicatrices d'un même groupe. En 1908-1909, le major Arthur Tremearne représente le visage très marqué d'un originaire de Kano, Awudu (90), qui dit porter les marques des Kutumbawa : trois yan baki ${ }^{20}$ (dessin aux commissures des lèvres) et deux grandes lignes jusqu'aux oreilles ${ }^{21}$, alors qu'en 1912 Fletcher décrit les marques des Kutumbawa comme étant un zubbe (dessin formé d'une série de lignes parallèles) avec des marques supplémentaires convergeant vers le nez (figure 1$)^{22}$.

Figure 1 - Dessins des marques Kutumbawa de Tremearne à gauche et de Fletcher à droite
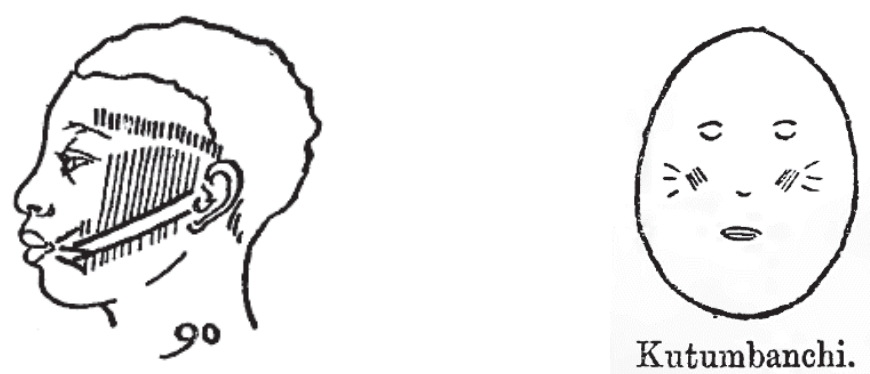

17. G. F. Lyon, A Narrative of Travels in Northern Africa in the Years 1818-1819 and 1820, op. cit., p. 228 ; D. Denham, H. Clapperton, W. Oudney, Discoveries in Northern and Central Africa in Years 1822, 1823, and 1824, op. cit., t. 1, p. 171.

18. J. Richardson, Narrative of a Mission to Central Africa Performed in the Years 1850-1851, op. cit., t. 2, p. 230.

19. R. Gaillard, L. Poutrin, «Étude anthropologique des populations des régions du Tchad et du Kanem », cité, t. 3, p. 22.

20. Tous les termes haoussas ou kanouris utilisés ici sont présents dans les sources du XIX ${ }^{\mathrm{e}}$ ou du début du $\mathrm{XX}^{\mathrm{e}}$ siècle. Le sens des mots a été vérifié dans George P. Bargery, A Hausa-English Dictionary and EnglishHausa Vocabulary, Londres, Oxford University press, 1934 ; Roy C. Abraham, Dictionary of the Hausa Language, Londres, University of London Press, 1968 (1946) ; Norbert Cyffer, John Hutchison (eds), Dictionary of the Kanuri language, Dordrech, Foris, 1990.

21. Major Arthur J. N. Tremearne, « Notes on Some Nigerian Tribal Marks », The fournal of the Royal Anthropological Institute of Great Britain and Ireland, 41, 1911, plate XXIII.

22. Roland S. Fletcher, Hausa Sayings and Folklore with a Vocabulary of New Words, Londres, Oxford University Press, 1912, p. 85. 
Certes, ces distorsions peuvent être dues au trait du dessinateur ou à des erreurs d'observation, mais certains éléments amènent à relativiser de telles hypothèses. Tremearne représente et répertorie les scarifications portées par une quarantaine d'individus se désignant tous comme étant des Haoussas originaires de Kano. Or il n'y en a pas deux qui portent la même marque. Trois exemples d'individus dont les deux parents sont désignés comme étant de Kano éclairent cette diversité (figure 2) ${ }^{23}$ : Adamu (5) porte cinq lignes sur la joue droite, six sur la gauche, un Bille, trois petites lignes entre les yeux et le nez, et des yan baki; Mohamma (46) porte un Kallango à l'extérieur de l'œil gauche, et Awudu (48) 3 yan uku uku sur le côté de chaque œil.

Figure 2 - Adamu (5), Mohamma (46) et Awudu (48) par Tremearne
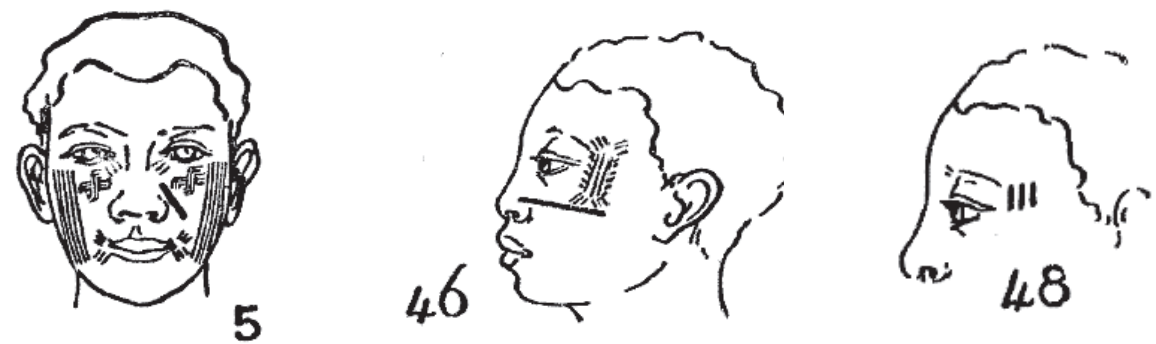

De plus, aucun de ces quarante individus ne porte de marque similaire à celle représentée cinquante ans plus tôt par Francis de Castelnau comme étant la marque des Haoussas de Kano (figure 3$)^{24}$.

Figure 3 - Mahammah par Castelnau portant à la bouche la marque des Haoussas de Kano

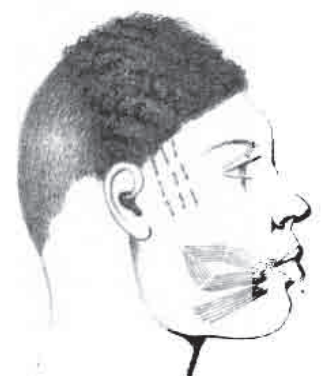

Ce constat d'extrême variabilité peut être poussé plus loin. Le nombre de cicatrices pour des dessins signalés par un même nom n'est pas forcément similaire. Un yan baki, marque constituée de plusieurs traits partant de chaque côté des commissures

23. Major A. J. N. Tremearne, « Notes on Some Nigerian Tribal Marks », art. cité, p. 163 et 166. Copyright (C) 1911 by John Wiley \& Sons, Inc. Reprinted by Permission of John Wiley \& Sons, Inc.

24. Francis de Castelnau, Renseignements sur l'Afrique centrale et sur une nation d'hommes à queue qui s'y trouverait, d'après le rapport des nègres du Soudan, esclaves à Babia, Paris, P. Bertrand, 1851, planche 2, figure 1. Cette marque pourrait être une forme particulière de yan baki. 
des lèvres, peut être formé de trois lignes de chaque côté de la bouche, mais aussi de quatre ou cinq, voire d'un dessin compliqué formé de plusieurs séries de traits ornementés. La configuration des incisions dépend des habitudes de celui qui les réalise, de son habileté et des usages locaux ou familiaux. Par ailleurs, une marque peut avoir été tracée, selon les lieux, pour des raisons différentes, tout en gardant le même nom. Un yar goshi (grande ligne sur le front), peut ainsi être désigné tantôt comme esthétique, tantôt comme médicinal. Enfin, porter des marques similaires ne signifie pas forcément être du même lieu ou appartenir au même groupe. Tremearne déplore que ces traces n'aient pas un caractère systématique et remarque que plusieurs tribus portent les mêmes marques ${ }^{25}$.

Ce qui frappe lorsque l'on observe ces dessins réalisés sur plus d'un siècle et demi (1820-2013), c'est l'évolution des représentations : les marques sont de moins en moins visibles, leur nombre diminue et les dessins se simplifient. De visages presque entièrement recouverts de deux ou trois types de scarifications différentes sur le front, les joues et le menton, on passe à des visages marqués le plus souvent sur les joues d'une ou de deux marques formées d'un nombre de traits moins important. Le contexte troublé du XIX ${ }^{\mathrm{e}}$ siècle, le Jihad, l'opposition à celui-ci et l'augmentation du trafic et de l'usage des esclaves nécessitaient de pouvoir être immédiatement identifié par ses alliés ou retrouvé par ses proches. Le contexte colonial a pour conséquence la diminution des conflits locaux et des razzias, et réduit d'autant la nécessité de porter son appartenance sur sa peau.

La pratique des scarifications évolue donc dans le temps mais aussi dans ses usages. D'une génération à l'autre, un même groupe peut abandonner ou modifier le dessin des marques qui le représentent, tandis que l'ensemble des membres d'un groupe ou des habitants d'une ville n'utilisent pas forcément une seule et même marque.

\section{Dessiner des archétypes, voir des individus}

Les dessins qui nous sont parvenus, figurant pour la plupart des marques désignées comme génériques sur des visages et des corps stéréotypés, témoignent d'un biais interprétatif lié au regard des voyageurs et des administrateurs européens qui les ont réalisés. Ce malentendu s'éclaire dans un dialogue autour d'un dessin qui a lieu dans le Sahara en 1822.

À son retour d'une exploration dans ce qui est aujourd'hui le Sud de la Libye, Lyon publie en 1821 son journal de voyage. Selon une pratique courante à l'époque, l'ouvrage contient, entre autres, une série de gravures des différents types physiques rencontrés sur sa route : Tuarick in a leather skirt, Tuarick of Ghat, Negresses of Sudan, Tibboo woman, Tibboo of Gatrone ${ }^{26}$. L'année suivante, les membres de la mission du Bornou, Denham, Clapperton et le docteur Walter Oudney emportent avec eux

25. Major A. J. N. Tremearne, « Notes on Some Nigerian Tribal Marks », art. cité, p. 162.

26. G. F. Lyon, A Narrative of Travels in Northern Africa in the Years 1818-1819 and 1820, op. cit., p. 110, 113, 182, $225,235$. 
sur le terrain l'ouvrage de Lyon qui leur sert aussi bien à identifier certains de leurs interlocuteurs qu'à préparer leur regard ou à faire sens de ce qu'ils voient. Sur la route de Mourzouk, lors d'une excursion, Oudney montre l'ouvrage aux marchands avec lesquels ils voyagent. À partir de là, le livre ne cesse de susciter la curiosité. Lorsqu'ils arrivent quelques mois plus tard dans la capitale du Bornou Kouka, ils sont assaillis de demandes chaque jour et plusieurs fois par jour : tout le monde veut voir le livre dont la réputation a précédé leur venue ${ }^{27}$. Ce n'est pas l'ouvrage en lui-même qui fascine, les manuscrits circulent régulièrement sur cette importante route transsaharienne, mais plutôt les gravures qu'il contient, et tout particulièrement celles de personnages, dans une région marquée par le tabou de la représentation humaine.

Denham, lui, présente l'ouvrage pour la première fois à Bilma dans la tente de Boo Kahloom, le représentant de Tripoli qui dirige l'escorte de deux cents hommes armés qui leur a été fournie par le sultan. Lorsque Boo Kahloom voit les gravures représentant des hommes et des femmes, il s'exclame avec enthousiasme qu'il reconnait tous les personnages représentés : « Celui-ci était l'esclave d'un tel, celui-ci était mon esclave $»^{28}$. Fasciné par ces dessins, il insiste sur le fait qu'il peut identifier chacun et loue pour cela le talent des Anglais. Ainsi, là où les observateurs européens voient des figures impersonnelles de types ethniques et où Lyon met en scène des archétypes anonymes censés représenter pour chaque tribu des manières caractéristiques de se vêtir et de se coiffer, Boo Kahloom, lui, reconnaît les individus qu'il a côtoyés.

Au-delà de son caractère anecdotique, cet exemple nous invite à questionner le regard porté par les observateurs européens sur les pratiques de scarifications. Les explorateurs, puis les administrateurs coloniaux ou les anthropologues dessinent les marques qu'ils voient sur un individu et lui demandent à quel groupe il appartient, mais leur regard et leur questionnement sont marqués par un schème conceptuel anticipatif qui associe scarification et ethnicité. Cette association est une évidence pour eux, mais l'analyse attentive des sources suggère une situation plus complexe.

\section{Du regard à l'enquête}

Lorsque l'observateur étranger ne se contente pas de quelques coups d'œil rapides, lorsque l'enquête se fait précise et la méthodologie systématique, des usages différenciés et complexes des pratiques de scarifications apparaissent. À cet égard,

27. D. Denham, H. Clapperton, W. Oudney, Discoveries in Northern and Central Africa in Years 1822, 1823, and 1824, op. cit., t. 1, p. 261 ; H. Clapperton, Difficult and Dangerous Roads: Hugb Clapperton's Travels in Sahara and Fezzan 1822-1825, édité par Jamie Bruce Lockhart et John Wright, Londres, Sickle Moon Books, 2000, p. 233-234.

28. D. Denham, H. Clapperton, W. Oudney, Discoveries in Northern and Central Africa in Years 1822, 1823, and 1824, op. cit., t. 1, p. 152 . 
l'une de nos sources se détache clairement des autres : il s'agit de l'enquête du major Tremearne sur les scarifications haoussas menée sur plus d'une centaine d'individus en 1908-1909. Anthropologue de formation et avocat de métier, cet Australien né en 1877 se passionne pour le folklore africain et occupe au début du $\mathrm{XX}^{\mathrm{e}}$ siècle plusieurs emplois civils en Afrique de l'Ouest britannique (Gold Coast, Sierra Leone, Nigeria), afin de poursuivre ses recherches sur la langue et la culture haoussa ${ }^{29}$.

La méthodologie de son enquête est similaire dans sa forme à une série d'autres enquêtes menées à travers le continent dans les années 1904-1915, notamment au Nyassaland par Hugh Stanus ou en Gold Coast sous l'égide de Cecil Hamilton Armitage $^{30}$. Dans l'introduction de son ouvrage, ce dernier donne les clés pour comprendre la généalogie de cette série d'études. Au début de l'année 1908, une circulaire du Secretary of State invite les officiers coloniaux britanniques d'Afrique de l'Ouest à répondre à un questionnaire transmis par le professeur Leo Frobenius à la veille de son expédition scientifique dans la région ${ }^{31}$. En 1904, avant son départ pour le Congo et la Deutsche Inner-Afrikanische ForschungsExpedition, le professeur Frobenius avait déjà transmis un questionnaire aux agents locaux du Kasaï, qui comprenait 79 questions sur les tribus africaines, et notamment sur les signes corporels portés par les populations (modifications des dents, tatouages, perforations des lèvres et du nez... $)^{32}$. Il semble que ce soit ce même questionnaire, ou un équivalent, que le professeur Frobenius ait transmis aux autorités coloniales britanniques ainsi qu'aux autorités françaises en 1908. Le publiciste Félix Dubois, alors au Soudan, raconte en effet l'arrivée d'une demande analogue à l'intention des administrateurs d'Afrique occidentale française : «Un beau jour, ceux-ci avaient reçu une brochure, imprimée, de vingt pages, ayant pour titre : Questionnaire de la Mission scientifique allemande. M. Leo Frobenius $\mathrm{y}$ demandait tout simplement de répondre à soixante-dix-neuf questions (avec sous-questions abondantes), [de] rédiger un vocabulaire, remplir des tableaux de tatouages et autres signes, transcrire des fables et des mythes indigènes, enfin accompagner le tout de photographies et de croquis. Pour comble, cet ensemble d'exigences visait non la seule peuplade au milieu de laquelle vivait le fonctionnaire

29. Il est nommé dans les années 1910 lecturer de haoussa et d'anthropologie à Cambridge. A. C. Haddon, «Obituary Major A. J. N. Tremearne », Folklore, 26 (4), 1915, p. 431.

30. L'enquête non publiée de Hugh Stannus a été étudiée dans M. Vaughan, « Scarification in Africa. Re-reading Colonial Evidence », art. cité, p. 391-395. Cecil H. Armitage, The Tribal Markings and Marks of Adornment of the Natives of the Northern Territories of the Gold Coast Colony, Londres, Royal Anthropological Institute of Great Britain and Ireland, 1924.

31. C. H. Armitage, The Tribal Markings and Marks of Adornment of the Natives of the Northern Territories of the Gold Coast Colony, op. cit., p. 2. Pour l'instant, le questionnaire n'a pas été retrouvé dans les archives britanniques ou nigérianes.

32. Ce document est conservé dans les archives belges. Charlotte Braillon, « Leo Frobenius et les tribus du bassin du Congo (1905-1906) : un questionnaire ethnographique oublié ? Histoire et genèse d'une représentation de l'Autochtone », communication au colloque «Leo Frobenius : histoire croisée de la constitution et l'appropriation d'un savoir ethnologique en France et en Allemagne », Francfort-sur-le-Main, 19-20 février 2014. 
sollicité, mais celui-ci était prié de se livrer au même travail sur encore deux autres peuplades de son voisinage. Un mois d'un labeur ininterrompu, pour le moins, voilà ce que M. L. Frobenius demandait à chacun, et pour... le roi de Prusse $»^{33}$. Il semble que dans certains cas le questionnaire ait été accompagné de fiches pré-remplies, comprenant le dessin d'un visage type, de profil et de face, et une série d'entrées à compléter : nom de l'individu, famille, tribu, genre, âge, nom du tatouage, style, couleur, totem du père, de la mère (figure 4$)^{34} \ldots$

\section{Figure 4 - Exemple de fiche conservée dans les archives Frobenius}

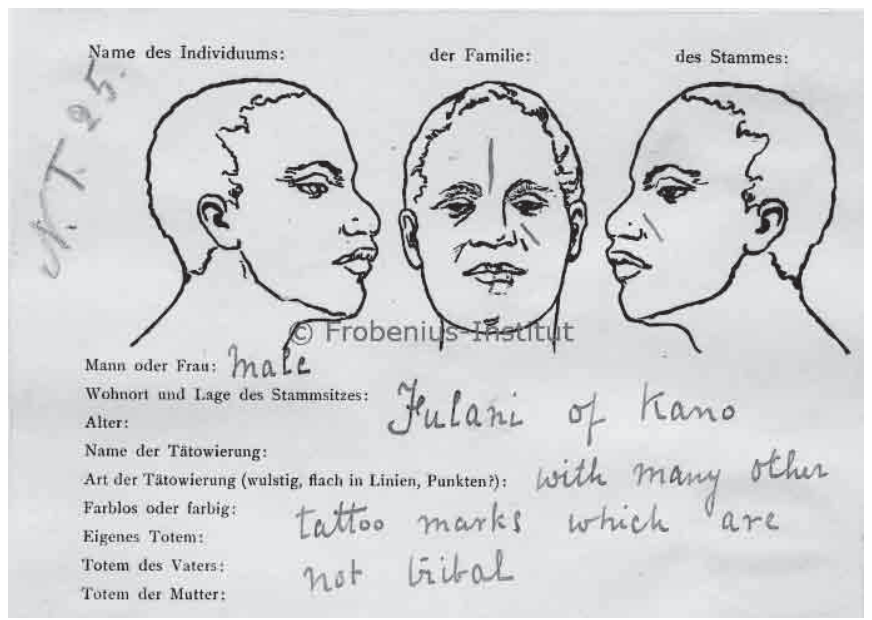

Bien qu'il ne désigne pas spécifiquement Frobenius et son questionnaire comme ayant motivé son enquête, Tremearne semble bien s'être saisi de ce programme. Les informations qu'il collecte sur chaque porteur de marques correspondent à la méthodologie proposée par Frobenius : pour chacun est indiqué son nom, le lieu de naissance de son père et de sa mère, le nom des marques quand elles en ont un, leur sens et les circonstances expliquant leur tracé 35 . De plus, Tremearne cite régulièrement les travaux de Frobenius dans ses ouvrages ${ }^{36}$.

33. Félix Dubois, Notre beau Niger..., Paris, Flammarion, 1911, p. 289-290.

34. Plusieurs séries de fiches sont conservées dans les archives Frobenius, complétées par différents individus à travers le continent. Pour la figure 4, le dessin a été réalisé au Cameroun entre 1910 et 1912, sans indication d'auteur. Archives de l'Institut Frobenius (Francfort-sur-le-Main), Kartei Bildarchiv 01265. Les fiches sont accessibles en lignes à partir du catalogue d'images numérisées de l'Institut Frobenius (http://bildarchiv. frobenius-katalog.de).

35. Les deux versions publiées de l'enquête de Tremearne ne sont pas identiques, ni dans les dessins ni dans les commentaires, certains détails étant alternativement notés dans l'une et pas dans l'autre. Major A. J. N. Tremearne, « Notes on Some Nigerian Tribal Marks », art. cité, p. 162-178 ; Major A. J. N. Tremearne, Hausa Superstitions and Customs: An Introduction to the Folklore and the Folk, Londres, John Bale \& Danielson, 1913, p. $518-535$.

36. Major A. J. N. Tremearne, Hausa Superstitions and Customs: An Introduction to the Folklore and the Folk, op. cit., p. 30, 113-114, 117, 138. 
C'est en 1908-1909, alors qu'il est en poste à Jemaan Daroro, province de Nassarawa dans le Nord du Nigeria, qu'il mène son enquête. Sur les 362 personnes qui se présentent à lui pour y participer, il n'en retient que 116 qu'il considère comme de véritables Haoussas, parce qu'elles parlent la langue, ont deux parents haoussas et sont reconnues comme telles par ses assistants ${ }^{37}$. Parmi elles, 15 ne portent pas de marques, 40 ont des marques sur le visage et le corps, 54 uniquement sur le visage et 7 uniquement sur le corps. Pour 29 d'entre elles, le porteur a indiqué les circonstances ou le sens du tracé : 10 sont médicinales, 7 indiquent un statut (2 la possession d'un troupeau, 2 la marque des bouchers et 3 le statut d'esclave), 6 sont de l'ordre du charme magique (2 pour obtenir de la nourriture, 3 pour attirer les femmes, la sixième est une marque de chance), 5 indiquent une appartenance géographique ou identitaire, une seule est décrite comme purement esthétique. La richesse des marques répertoriées et la diversité des fonctions évoquées semblent montrer qu'un même individu porte sur sa peau des marques aux fonctions très variées, réalisées à différents moment de sa vie. Ce constat nous invite à affiner l'analyse et à tenter de reconstituer à l'échelle de la vie d'un individu les moments et les raisons qui occasionnent des pratiques de scarification.

\section{Marquer l'appartenance, se soigner, s'embellir}

Au XIX ${ }^{\mathrm{e}}$ siècle $^{38}$, dans la majorité des cas, un enfant reçoit ses premières scarifications tout de suite après sa naissance. La plupart du temps, celles-ci sont réalisées par un praticien professionnel appelé wanzam en haoussa, wanzamma en kanouri, wandjam en songhaï ${ }^{39}$. Dans son dictionnaire kanouri/anglais de 1854, Sigismund Koelle donne la définition suivante du wontsam : « Sorte de barbier dont le travail est de raser la tête des hommes, de tatouer les enfants au huitième jour de leur vie et de circoncire plus tard les jeunes garçons à cinq, sept, neuf, onze ou treize ans $\gg^{40}$. Le mot wanzam (barbier) est également présent dans le récit de Barth et dans le dictionnaire haoussa du linguiste James Frederick Schön de $1888^{41}$. Dans son récit de vie recueilli en 1949, Baba de Karo, née en 1890, décrit cette

37. Major A. J. N. Tremearne, « Notes on Some Nigerian Tribal Marks », art. cité, p. 162.

38. Il semble que la pratique des scarifications soit très ancienne dans certaines régions du Niger actuel. Les statuettes anthropomorphes datant du $\mathrm{III}^{\mathrm{e}}$ au XIII ${ }^{\mathrm{e}}$ siècle retrouvées par Boubé Gado portent toutes des dessins de scarifications faciales. Boubé Gado, « Un "village des morts” à Bura en République du Niger », dans Vallées du Niger, catalogue de l'exposition, Paris, Réunion des musées nationaux, 1993, p. 369.

39. Joseph H. Greenberg, « Linguistic Evidence for the Influence of the kanuri on the Hausa », The fournal of African History, 1 (2), 1960, p. 209.

40. Sigismund Koelle, African Native Literature, or Proverbs, Tales, Fables and Historical Fragments in the Kanuri or Bornu Language, to Which Are Added a Translation of the Above and a Kanuri-English Vocabulary, Londres, Church Missionary House, 1854, p. 427.

41. H. Barth, Travels and Discoveries in North and Central Africa. Being a Fournal of an Expedition Undertaken under the Auspices of H.B.M.'s Government, in the Years 1849-1855, op. cit., t. 2, p. 310 ; James Frederick Schön, Appendix to the Dictionary of the Hausa Language: Hausa-English Part, with Additions of Hausa Literature, Londres, Church Missionary House, 1888, p. 167-168. 
pratique. D'après ce qu'elle a observé, c'est au troisième jour pour le garçon et au quatrième pour la fille que le barbier pratique les scarifications et circoncit par la même occasion les garçons ${ }^{42}$. Au $\mathrm{XX}^{\mathrm{e}}$ siècle, dans les communautés musulmanes, les scarifications sont souvent réalisées lors de la cérémonie du nom, au cours de laquelle le nouveau-né est rasé, nommé, scarifié et parfois aussi circoncis ${ }^{43}$. Dans les groupes non musulmans asna du Niger et maguzawa du Nord Nigeria, c'est aussi un wanzam musulman qui pratique circoncision et scarification mais cette fois sans cérémonie ${ }^{44}$. Ces premières scarifications font partie d'un ensemble de rites qui introduisent l'enfant au sein du groupe social, selon des usages très variables, mais partagés par les musulmans et les non-musulmans. Mais de quel type de groupe est-il précisément question ?

Si l'on ne retient que les lectures antérieures à la période coloniale, le critère géographique semble déterminant. Pour l'ambassadeur tripolitain Abderrahman Aga et son valet haoussa interrogé par Carsten Niebuhr à Copenhague en 1772, les scarifications permettent de reconnaître le pays d'origine (Vaterland), pour Clapperton, de connaître immédiatement le pays (country) de celui qui les porte, et pour Richardson, elles désignent les nations particulières des noirs ${ }^{45}$. Certes, le terme de nation est tiré du vocabulaire politique européen, mais Richardson a raison de souligner ainsi l'importance du politique.

Comme le montrent de nombreux exemples dans les sources, cette appartenance n'est pas déterminée mécaniquement ou systématiquement héritée des parents. Un enfant peut porter les marques du groupe ou de l'origine de ses parents, mais aussi celles du lieu où il est né, même si ses parents viennent d'ailleurs. Selon Castelnau, « bien que le père de Braz soit de Zozo [Zaria], il est né à Java, à trois journées de marche de cette ville, et il porte sur la figure les marques tatouées des habitants de cette partie $\gg^{46}$. Baba de Karo raconte, elle aussi, comment ses parents, après avoir quitté le Bornu en raison d'un conflit familial, ont abandonné

42. Mary Smith, Baba of Karo: A Woman of the Muslim Hausa, Londres, Faber and Faber, 1954, p. 139.

43. Ismail Abdalla, Islam, Medicine, and Practitioners in Northern Nigeria, Lewiston, E. Mellen Press, 1997, p. 127.

44. Guy Nicolas, Dynamique sociale et apprébension du monde au sein d'une société hausa, Paris, Muséum national d'Histoire naturelle, 1975, p. 85, Murray Last, « The Presentation of Sickness in a Community of Non-Muslim Hausa », dans Joseph B. Loudon, Social Anthropology and Medicine, San Francisco, Academic Press, 1976 , p. 128. 45. Carsten Niebuhr, « Das Innere von Afrika », Neue Deutsches Museum, 1790, p. 981 ; D. Denham, H. Clapperton, W. Oudney, Discoveries in Northern and Central Africa in Years 1822, 1823, and 1824, op. cit., t. 2, p. 144 ; J. Richardson, Narrative of a Mission to Central Africa Performed in the Years 1850-1851, op. cit., t. 2, p. 115. Les observateurs coloniaux, eux aussi, ont souvent eu cette lecture géographique sans se défaire pour autant du biais ethnique. Pour Georg Merrick, ce sont des marques tribales (tribal marks) par lesquelles chacun porte le nom de son district sur la peau. Captain Georg Merrick, Hausa Proverbs, Londres, Trübner and Co., 1905, p. 93. Pour Gilbert Vieillard « Les marques tribales, cicatrices du visage et du corps, qui se font encore traditionnellement et auxquelles on reconnaît l'origine des individus, pourraient être étudiées, mais le plus souvent elles paraissent être devenues plus géographiques qu'ethniques ». Gilbert Vieillard, « Coutumier du cercle de Zinder 1932 », Coutumiers juridiques de l'Afrique occidentale française, Paris, Larose, 1939, t. 3 , p. 112.

46. F. de Castelnau, Renseignements sur l'Afrique centrale et sur une nation d'hommes à queue qui s'y trouverait, d'après le rapport des nègres du Soudan, esclaves à Babia, op. cit., p. 28. 
les scarifications de cette région et ont adopté celles de leur lieu d'immigration ${ }^{47}$. À l'inverse, certains groupes continuent de pratiquer des scarifications liées à une origine ancienne même plusieurs générations après avoir émigré ${ }^{48}$. Certains enfants dont les parents sont originaires de deux lieux différents, voire de deux groupes différents, peuvent porter sur leur visage une combinaison de cette origine double, comme Mohamma, qui a, autour de la bouche, la marque des Haoussas de Kano et, sur les tempes, des marques pratiquées dans l'Aïr ${ }^{49}$. À Zinder, ville particulièrement cosmopolite au $\mathrm{XIX}^{\mathrm{e}}$ siècle, cette pratique est institutionnalisée : sur la joue droite, les marques du père, sur la gauche, celles de la mère ${ }^{50}$. Parfois, les marques ne correspondent ni à l'origine du père ni à celle de la mère. C'est le cas de trois des interlocuteurs de Tremearne qui évoquent l'origine géographique ou identitaire de leurs marques. Alhassan (7) porte une marque peule mais dit ne pas être peul, Umoru (58) déclare porter une marque Buzu comme son grandpère et Musa (80) une marque Wangara mais ses deux parents sont de Bauchi ${ }^{51}$. L'enfant porte parfois des scarifications liées à un lien d'amitié que ressent sa mère ou son père pour un membre d'un autre groupe. Tremearne évoque cette pratique sous le terme d'abuiya à propos de Kumatu $(11)^{52}$.

L'appartenance symbolisée par les scarifications n'est donc pas héritée mécaniquement, mais choisie parmi un éventail de possibilités et en fonction de critères qui ne sont pas, a priori, systématiquement déterminés. Dans ces régions, chacun possède de manière cumulative plusieurs identités qui se superposent : celles héritées des parents, celles acquises par la résidence, la migration ou le mariage. Toutes ces identités, acquises au cours de l'histoire ou d'un parcours individuel, sont maintenues afin de maximiser les possibilités d'alliance et de collaboration et sont mobilisées différemment selon les lieux et les circonstances ${ }^{53}$.

Toutefois, cette capacité à mobiliser différentes identités et à choisir les scarifications que l'on fait porter à ses enfants a ses limites. Une erreur, le non-respect des règles tacites qui encadrent cette pratique ou l'adoption de scarifications

47. M. Smith, Baba of Karo: A Woman of the Muslim Hausa, op. cit., p. 37.

48. Captain G. Merrick, Hausa Proverbs, op. cit., p. 94 ; P. Lovejoy, Caravans of Kola: The Hausa Kola Trade, 17001900, op. cit., p. 84-85.

49. F. de Castelnau, Renseignements sur l'Afrique centrale et sur une nation d'bommes à queue qui s'y trouverait, d'après le rapport des nègres du Soudan, esclaves à Babia, op. cit., p. $6 \mathrm{~s} 1$.

50. Maurice Abadie, La colonie du Niger, Paris, Société d'éditions géographiques, maritimes et coloniales, 1927, p. 220.

51. Major A. J. N. Tremearne, « Notes on Some Nigerian Tribal Marks », art. cité, p. 164, 166-167, 168 ; A. J. N. Tremearne, Hausa Superstitions and Customs: An Introduction to the Folklore and the Folk, op. cit., p. 520, $524,526$.

52. Major A. J. N. Tremearne, « Notes on Some Nigerian Tribal Marks », art. cité, p. 164 ; A. J. N. Tremearne, Hausa Superstitions and Customs: An Introduction to the Folklore and the Folk, op. cit., p. 520. Lors de mon terrain au Niger en septembre 2013, certains de mes interlocuteurs ont évoqué cette pratique ; ainsi, une mère songhaï peut donner des scarifications peules à son enfant en raison de l'amitié qu'elle ressent pour une amie peule qui a accouché la même semaine.

53. Benedetta Rossi, « Being and Becoming Hausa in Ader (Niger)», dans Benedetta Rossi, Anne Haour, Being and Becoming Hausa: Interdisciplinary Perspectives, Leyde, Brill, 2010, p. 135-136. 
auxquelles on ne peut prétendre peuvent aboutir, dans certaines circonstances, au rejet de l'enfant. En dépit de leur diversité, les traditions orales sur les origines des Maouris comportent toutes un passage sur l'origine de leurs cicatrices ${ }^{54}$. Les Maouris seraient issus de l'union d'un étranger musulman venu de l'Est et d'une autochtone animiste. Lorsque leur enfant naît, son père est absent, mais la famille de la mère souhaite lui faire porter les mêmes scarifications que ce dernier. Dans plusieurs versions, la famille de la mère prend comme modèle les marques portées par le représentant envoyé par le père, mais celui-ci est un esclave qui ne porte pas les mêmes marques que son maître. Dans toutes les versions, cette erreur entraîne le rejet de l'enfant par son père.

Au cours de sa vie, un individu se retrouve régulièrement entre les mains d'un wanzam qui marque son corps et son visage de nouvelles cicatrices. Les wanzam dispensent des soins thérapeutiques qui consistent en des interventions de petite chirurgie, notamment des saignées pour excès de sang ou pour évacuer le mauvais sang qui, en dehors de la circoncision, représentent la part la plus importante de leur pratique ${ }^{55}$. Pour protéger son enfant ou se protéger soi-même, pour attirer la chance ou soigner une agression (magique ou humaine), des mutilations thérapeutiques ou prophylactiques sont pratiquées, la plupart du temps à titre préventif. Les lésions sont réalisées loco dolenti, c'est-à-dire à l'endroit où devrait se situer la douleur ou le mal que l'on craint : une ligne sur le front pour prévenir les maux de tête comme pour Mohamma (31) ou empêcher une hémorragie comme pour Ahmadu (55), des incisions sur l'abdomen pour soulager des maux de ventre comme pour Baba (44) ou Ibrahim (72) ou autour des yeux pour soigner une conjonctivite comme pour Awudu (112) ${ }^{56}$. La médecine ainsi pratiquée est personnelle et la maladie est envisagée comme concernant spécifiquement un individu. Ainsi, chacun utilise des charmes pour se protéger, et si cette protection échoue, le traitement est l'affaire de l'individu ${ }^{57}$. Ces pratiques de saignées thérapeutiques semblent mises en œuvre au XIX ${ }^{\mathrm{e}}$ siècle par l'ensemble des populations du Sahara et du Sahel central, des Touaregs de l'Aïr aux Songhaïs du fleuve Niger, en passant par les populations du lac Tchad ou les Toubous du Tibesti.

Sur son visage et sur son corps, un individu porte différentes séries de marques réalisées à différents moments de sa vie : des marques d'appartenance affirmant une identité attribuées dans sa petite enfance et des marques liées à son histoire personnelle et familiale, aux maladies qu'il a eues ou qu'il a craint d'avoir, aux charmes qu'il a souhaité utiliser. Il peut aussi choisir de porter des marques

54. Pour ces différentes versions, voir Mahamane Karimou, Les Mawri zarmaphones, Niamey, IRSH, 1977, p. $19-20$.

55. I. Abdalla, Islam, Medicine, and Practitioners in Northern Nigeria, op. cit., p. 128.

56. Major A. J. N. Tremearne, « Notes on Some Nigerian Tribal Marks », art. cité, p. 165, 166, 167, 170 ; A. J. N. Tremearne, Hausa Superstitions and Customs: An Introduction to the Folklore and the Folk, op. cit., p. 522, $524,526,528$

57. M. Last, « The Presentation of Sickness in a Community of Non-Muslim Hausa », cité, p. 127. 
purement esthétiques liées à des modes, par définition passagères, ou s'approprier des dessins vus sur d'autres ${ }^{58}$. Certains portent un symbole lié à leur profession ou à leur statut, mais ce n'est pas systématique. D'une génération à l'autre, ces marques peuvent changer et un individu ne reproduira pas forcément celles de son visage et de son corps sur ses propres enfants.

\section{Signe distinctif mais pas signe de distinction}

Dans les sociétés étudiées ici, au XIX ${ }^{\mathrm{e}}$ siècle, on marque sa différence sociale, religieuse et de genre par son apparence, notamment sa coiffure et son vêtement. Or, en matière de scarifications, ces trois critères ne sont pas systématiquement déterminants. Hommes et femmes, mais aussi nobles et esclaves portent parfois les mêmes marques, alors qu'ils ne sauraient être vêtus de manière similaire ${ }^{59}$. Baba de Karo raconte que les enfants d'esclaves nés dans sa famille portent les mêmes cicatrices que les autres ${ }^{60}$. Enfin, dans des sociétés religieusement très polarisées, notamment entre ceux qui suivent le Jihad et ceux qui le rejettent, les scarifications faciales ne semblent pas être un sujet de désaccord.

Dans leurs prêches et leurs ouvrages, les Jihadistes invitent à montrer par le vêtement qui l'on est et ce que l'on défend ${ }^{61}$. Or, à notre connaissance, parmi leurs nombreux écrits, il n'existe qu'une seule mention négative des scarifications qui est l'œuvre de Usman dan Fodio dans son ouvrage Ihyā al-sunna wa-ikhmād al-bid'a datant de $1793^{62}$. L'auteur y dénonce la pratique des modifications corporelles ornementales féminines en reprenant l'un des passages du Tafsìr d'al-Bukhārī consacré au tatouage : «D'après 'Abdallâh (Ibn Mas'ûd), Dieu a maudit les femmes qui se tatouent, celles qui se font tatouer, celles qui s'épilent le visage et celles qui espacent leurs dents par coquetterie, dénaturant ainsi les choses créées par Dieu. "Pourquoi, moi, ajouta 'Abdallâh, ne maudirais-je pas ceux que le Prophète a maudits et qui sont maudits par le Livre de Dieu qui a dit 'Ce que vous donne

\footnotetext{
58. La part de ces modes est quasi impossible à établir pour le XIX ${ }^{\mathrm{e}}$ siècle, mais il est clair qu'une part importante des marques portées aujourd'hui, en particulier chez les femmes, leur sont liées. À titre d'exemple, dans les années 1990 la mode des yan baki s'est très largement diffusée sur l'ensemble du Niger (Agadez, Arlit, Niamey, Zinder) chez les jeunes filles non encore mariées. Enquête à Niamey (Niger), septembre 2013.

59. Mahdi Adamu, The Hausa Factor in West African History, Ibadan, Oxford University Press Nigeria, 1978, p. 188.

60. M. Smith, Baba of Karo: A Woman of the Muslim Hausa, op. cit., p. 43.

61. Comme le dit Nana Asmau, la fille d'Usman dan Fodio, dans un poème en ajami (graphie arabe) haousa et fufulde, publié et traduit dans Beverly B. Mack, Jean Boyd, One woman's jibad: Nana Asma'u, scholar and scribe, Bloomington, Indiana university press, 2000, p. 158.

62. Ismail Ayinla Babatunde Balogun, «A Critical Edition of Ihyā' al-sunna wa-ikhmād al-bid'a of Uthman b. Fudi Popularly Known as Usumanu dan Fodio », Ph. D., University of London, 1967, p. 364. Il n'existe pas à ce jour de traduction de ce texte, mais simplement une édition critique en arabe.
} 
l'envoyé, prenez-le' (sourate 59, verset 7)' $\gg^{63}$. En revanche, les mutilations thérapeutiques ou prophylactiques, notamment les saignées, sont encouragées par Usman dan Fodio ainsi que par son fils, Muhammad Bello ${ }^{64}$. Cette différence de traitement correspond aux traditions islamiques prophétiques : le tatouage dans un but esthétique n'apparaît pas dans le Coran mais il est dénoncé dans les badîth, tandis que la saignée y est largement recommandée. Le regard fondamentaliste des leaders du Jihad sur la sacralité du corps humain, regard marqué par les théories des premiers temps de l'islam, ne s'applique pas strictement à la question des scarifications, certainement en raison de la part importante des usages thérapeutiques de cette pratique dans le Sahel central au XIX ${ }^{\mathrm{e}}$ siècle.

Au demeurant, il semble que l'appréciation négative des scarifications ornementales dépasse le cadre strictement religieux. Au XIX siècle, dans l'espace haoussaphone, le langage joue un rôle essentiel au quotidien et les activités journalières sont rythmées par des récits et des jeux de langage. Au sein de cette oralité mémorisée, des contes ou des fables sont récités au cours de performances verbales réalisées en groupe. Ces récits ont souvent pour protagonistes des animaux se comportant comme des hommes. Le propos, rarement didactique, a plus vocation à amuser qu'à instruire, mais concerne des problèmes caractéristiques des sociétés locales ${ }^{65}$. En 1856, à Londres, Dorugu, le compagnon de voyage de Barth, raconte à Schön un conte haoussa à propos d'une hyène, d'un renard et de poissons, qui moque ceux qui se scarifient pour des raisons esthétiques ${ }^{66}$. Le renard attrape une grande quantité de poissons qu'il propose à la hyène de partager avec lui, mais celle-ci les dévore presque tous, et cela le met en colère. C'est alors que la hyène voit une pintade dont elle admire le dos décoré, et elle demande qui pourrait lui faire d'aussi beaux dessins. Le renard lui répond « amène-moi un couteau et je te les ferai ». La hyène s'exécute et le renard lui balafre le dos en se moquant : « Tu as mangé tous mes poissons, j'ai scarifié ton dos $\gg^{67}$. Le renard se venge en exploitant

63. Muhammad ibn Ismā‘ìl al- Bukhārī, Le Sahîh al-Bukbârî. Tome 4, Hadiths 5475-7563, traduit par Octave Houdas et William Marçais, entièrement revu, corrigé et annoté par Corentin Pabiot, Paris, Maison d'Ennour, 2007, p. 195. Titre 77 - Des vêtements -, chap. 82 - Des femmes qui créent un espace entre leurs dents par coquetterie.

64. 'Abdullah Hakim Quick, Aspect of Islamic Social Intellectual History in Hausaland Uthman ibn Fudi 17741804 C.E., Ph. D., University of Toronto, 1995, p. 127 ; I. A. B. Balogun, « A Critical Edition of Ihyā' al-sunna $w a-i k h m \bar{a} d$ al-bid'a of Uthman b. Fudi Popularly Known as Usumanu dan Fodio », cité, p. 260-262.

65. Michael Garfield Smith, «Foreword», dans Frank Edgar, Neil Skinner, Hausa Tales and Traditions: An English Translation of Tatsuniyoyi na Hausa, Londres, Franck Cass, 1967 (1911), t. 1, p. XIII.

66. James Frederick Schön, Charles Henry Robinson, Magana Hausa. Hausa Stories and Fables, Londres, Society for promoting Christian knowledge, 1906, p. 106-107 ; J. F. Schön, Appendix to the Dictionary of the Hausa Language: Hausa-English Part, with Additions of Hausa Literature, op. cit., p. 212-213. Il existe plusieurs variantes de ce conte recueillies au début du $\mathrm{XX}^{\mathrm{e}}$ siècle qui mentionnent notamment une araignée à la place du renard. Robert Sutherland Rattray, Hausa Folklore, Customs and Proverbs etc. Collected and Transliterated with English Translation and Notes, Oxford, The Clarendon Press, 2 (27), 1913, p. 74-76 ; A. J. N. Tremearne, Hausa Folk-Tales: The Hausa Text of the Stories in Hausa Superstitions and Customs, in Folklore, and in Other Publications, Londres, J. Bale, Sons \& Danielsson, 36, 1914, p. 88-89.

67. Le mot utilisé pour évoquer les marques réalisées sur le dos de la hyène est sausawa/shaushawa qui désigne en haoussa les scarifications faciales et corporelles chez les êtres humains. 
la vanité de la hyène symbolisée par le fait de vouloir sur son propre corps des dessins similaires à ceux vus sur un autre.

Les pratiques de scarification ne reflètent pas mécaniquement les grandes distinctions qui organisent au XIX ${ }^{\mathrm{e}}$ siècle les sociétés du Sahel central : jihadistes et opposants au Jihad, musulmans et non-musulmans, hommes et femmes, esclaves et nobles, tous en portent sans qu'elles traduisent forcément ces oppositions. Ce qui peut se lire sur la peau est donc le reflet d'autres enjeux.

\section{N'étaient ses marques je ne pourrais le reconnaître}

L'espace du Sahel central au XIX ${ }^{\mathrm{e}}$ siècle est caractérisé par une très grande mobilité des individus et des groupes. Les voyageurs, les marchands itinérants, les pèlerins, les éleveurs, les paysans, les esclaves, les enseignants et les étudiants parcourent régulièrement aussi bien des petites distances que des centaines de kilomètres, quittent leurs lieux de résidence, parfois pendant plusieurs mois, voire des années, dans d'immenses caravanes ou en petits groupes, plus rarement seuls. Dans cette société en mouvement constant, la plupart des communautés villageoises et urbaines sont cosmopolites et constituées d'éléments disparates, notamment d'immigrants venus d'horizon divers. Cette région est également marquée par l'importance de l'esclavage. Au milieu du XIX siècle, dans le sultanat de Sokoto, les esclaves représentent environ $50 \%$ de la population ${ }^{68}$.

Dès lors, dans ce monde marqué par une très grande mobilité géographique et sociale, une forte urbanisation, et où règne le risque d'être capturé sur les routes, le fait de posséder des preuves de son identité et de pouvoir être reconnu peut sauver un individu de la capture, lui permettre d'être retrouvé par sa famille, d'être réintégré au sein de son groupe ou de faire valoir ses droits. Combinées au vêtement et à la coiffure, les marques tracées sur le visage et sur le corps permettent à l'observateur averti de repérer en un clin d'œil d'où vient celui qu'il a en face de lui, s'il fait partie de son groupe ou d'un groupe $\mathrm{ami}^{69}$. Cela est particulièrement précieux sur un champ de bataille ou en cas de raid esclavagiste ${ }^{70}$, mais les sources révèlent encore d'autres formes d'identification liées aux scarifications.

68. Selon l'estimation proposée par Barth à partir de ses observations pour la région de Kano dans les années 1850. H. Barth, Travels and Discoveries in North and Central Africa. Being a Fournal of an Expedition Undertaken under the Auspices of H.B.M.'s Government, in the Years 1849-1855, op. cit., t. 1, p. 510, 523 ; P. Lovejoy, Transformations in Slavery. A History of Slavery in Africa, Cambridge, Cambridge University press, 2000, p. 193 ; H. J. Fisher, Slavery in the History of Muslim Black Africa, Londres, Hurst, 2001, p. 33.

69. Nachtigal évoque son guide capable d'identifier instantanément l'origine de tous ceux qu'il voit au marché de Kouka. A. G. B. Fisher, H. J. Fisher, Sahara and Sudan. Volume II. Sahara and Sudan, Kawar, Bornu, Kanem, Borku, Ennedi, Londres, Hurst \& Company, 1980, p. 221.

70. C'est l'usage attribué aujourd'hui localement aux pratiques de scarification. Aucune source du XIX ${ }^{\mathrm{e}}$ siècle ne formule spécifiquement les choses en ces termes, mais les traditions orales tout comme les résultats des enquêtes sur ces questions sont unanimes. M. Karimou, Les Mawri zarmaphones, op. cit., p. 36 ; Pierre Donaint, Les cadres géographiques à travers les langues du Niger : contribution à la pédagogie de l'étude du milieu, Niamey, Institut nigérien de recherches en sciences humaines, 1975, p. 248 ; Enquête à Niamey, septembre 2013. 
Dans son autobiographie recueillie en haoussa par Schön, Dorugu raconte qu'après l'enlèvement et l'asservissement de sa mère (nous sommes alors dans les années 1840) son père a décidé de confier son frère âgé de 6 ou 7 ans à l'un de ses amis pour qu'il l'élève. Il était alors courant pour un parent de se séparer d'un ou de plusieurs de ses jeunes enfants et de les confier à des proches. Dix ans plus tard, Dorugu évoque avec tristesse cette séparation et explique que s'il revoyait son frère il ne pourrait le reconnaître que grâce à ses scarifications (shaushawa $)^{71}$. Cette reconnaissance d'un enfant perdu de vue par la lecture des marques ne semble d'ailleurs pas forcément limitée aux scarifications, comme le montre un conte, écrit en ajami haoussa et recueilli au début du $\mathrm{XX}^{\mathrm{e}}$ siècle $^{72}$. Une petite fille est chassée du foyer par son frère, fils d'une autre épouse de son père, qui la laisse pour morte dans la forêt. Elle est alors recueillie par un riche marchand itinérant (fatake) qui l'élève comme sa fille. Devenue une jeune femme, elle est demandée en mariage par un homme qui se révèle être ce frère qui l'a chassée enfant. Elle le reconnaît, mais lui ne la reconnaît pas. Ils se marient, mais elle se refuse à lui sans lui dire pourquoi. Un jour, alors qu'elle est seule chez elle, elle chante ses malheurs, une vieille femme du village l'entend et raconte tout à ses parents. Son père la convoque et lui demande si elle est bien sa fille disparue, mais elle refuse de répondre. Il lui dit alors « si tu es ma fille, tu as dans le dos une brûlure que tu as eue enfant » et il lui ordonne de se dévêtir. Elle s'exécute, révélant ainsi son identité, et réintègre le foyer de ses parents, tandis que son frère et mari dont la traîtrise est révélée se suicide ${ }^{73}$. Dans les deux cas, un parent reconnaît un proche perdu de vue alors qu'il était enfant et dont la physionomie a trop changé pour pouvoir être identifiée, en lisant des signes sur son visage ou sur son corps.

Cette identification par la description du corps et par la vue est présente dans un autre conte haoussa, intitulé « Le chef » recueilli au début du $\mathrm{XX}^{\mathrm{e}}$ siècle et consacré à la question du rachat des individus razziés ${ }^{74}$. Dans cette histoire, un maguje (non-musulman) et un mallam (lettré musulman), tous deux rentrant de voyage, constatent à l'arrivée dans leur village que celui-ci a été attaqué et que leurs enfants respectifs ont été enlevés. Ils partent alors à leur recherche et arrivent au bout de quelques jours dans le village du chef qui les a capturés. Là, chacun

71. Il ajoute que son frère, lui, ne pourrait l'identifier que s'il lui disait quelque chose lui rappelant leur parenté. J. F. Schön, C. H. Robinson, Magana Hausa. Hausa Stories and Fables, op. cit., p. 7. On trouve une traduction annotée en anglais d'une partie de l'ouvrage en haoussa de J. F. Schön, C. H. Robinson dans Anthony Kirk-Greene, Paul Newman, West African Travels: Two Autobiographical Narratives from Northern Nigeria, New Haven/Londres, Yale University Press, 1971, p. 34.

72. R. S. Rattray, Hausa Folklore, Customs and Proverbs etc. Collected and Transliterated with English Translation and Notes, op. cit., 2 (2), p. 50-66, notamment p. 62-64. On retrouve un motif semblable dans les contes russes, mais, si le thème est proche, la fonction de ce motif est différente. Dans les contes russes, la marque a été imposée par la princesse dans le cadre de l'histoire et symbolise l'admission du futur époux dans le clan de la femme. Vladimir Ja. Propp, Les racines historiques du conte merveilleux, Paris, Gallimard, 1983 (1946), p. 396-402.

73. Ce conte est marqué par un thème récurrent de la littérature orale haoussa, à savoir la jalousie entre frères et sœurs de mères différentes dans un contexte de polygamie.

74. F. Edgar, N. Skinner, Hausa Tales and Traditions: An English Translation of Tatsuniyoyi na Hausa, op. cit., p. $170-173$. 
explique qu'il cherche son enfant et le décrit physiquement. Le maguje mentionne en particulier la hernie ombilicale de son fils. Le chef met alors séparément le maguje et le mallam en présence d'un groupe d'esclaves et demande à chacun s'il reconnaît son enfant. Chacun identifie le sien par des signes distinctifs et, après quelques péripéties, le rachète.

Le rôle de la description des signes sur la peau comme preuve de l'identité apparaît également dans une anecdote rapportée en 1772 à Carsten Niebuhr par Abderrahman Aga, ambassadeur de Tripoli à Copenhague. Il lui raconte comment il est interpellé, alors qu'il vient d'acheter une jeune esclave, par une autre de ses esclaves qui, dès qu'elle voit la jeune fille, s'exclame qu'elle est sa compatriote et peut-être même sa fille qui a été enlevée quinze ans plus tôt lorsqu'elle était enfant $^{75}$. Pour le prouver, elle décrit les marques qui lui avaient été faites sous la poitrine et entre les épaules. Quelqu'un examine alors le corps de la jeune fille aux endroits indiqués, et constate qu'elle porte bien les marques spécifiées, celle-ci est donc reconnue comme sa fille.

Dans ces différentes histoires, les marques évoquées par les proches au moment de la reconnaissance ne sont pas liées à l'identité, mais plutôt à des usages familiaux ou à des histoires individuelles. Elles ne sont pas non plus immédiatement visibles et seul un membre de la famille peut les (re)connaître et les faire valoir comme preuve. La lecture des signes sur la peau semble donc s'opérer à deux niveaux, l'un, public, tourné vers les autres, qui atteste une identité souvent liée au politique, l'autre, caché, où la marque ne peut être déchiffrée que par ceux qui ont partagé l'intimité de la personne. Les scarifications apparaissent ainsi, même lorsque ce n'est pas leur but premier, comme dans le cas des pratiques thérapeutiques, comme des moyens de répondre à la nécessité sociale d'identifier les individus. Elles ne sont pas normées et n'obéissent pas à des règles strictes. Elles ne sont pas non plus le reflet d'un savoir d'État, mais plutôt d'un savoir social dont une partie est immédiatement déchiffrable par tous et une autre accessible seulement à ceux qui partagent avec le porteur une expérience intime.

Néanmoins, elles ne sont pas moins modifiables ou moins instrumentalisables que les papiers d'identité utilisant les technologies de l'écrit. Les scarifications ne sont pas définitives, elles disparaissent parfois au cours de la vie et peuvent être modifiées par leurs porteurs ${ }^{76}$. El Hadj Ahmed ben Mohammed el-Fellati, pèlerin peul dont le témoignage a été recueilli par des agents français en Tunisie lors de son deuxième voyage pour La Mecque en 1892, raconte ainsi à propos de son séjour à Abuja, ville qui justement résiste à la domination peule : «Je ne fus pas mal reçu en raison de ma couleur très foncée et des marques que je porte sur

75. C. Niebuhr, « Das Innere von Afrika », cité, p. 982.

76. Notamment Bello (47) ou Musa (56). Major A. J. N. Tremearne, « Notes on Some Nigerian Tribal Marks », art. cité, p. 166 ; A. J. N. Tremearne, Hausa Superstitions and Customs: An Introduction to the Folklore and the Folk, op. cit., p. 524. 
mon visage, et grâce auxquelles il m'est facile de me faire passer pour un simple Bornouan non foullane $\gg^{77}$. Des groupes entiers peuvent jouer parfois du caractère instrumentalisable de ces marques. Ainsi, à la fin du XIX ${ }^{\mathrm{e}}$ siècle, certaines familles musgum Kalang de Guirvidig décident de scarifier leurs enfants sur le modèle bornouan soit pour leur éviter d'être capturés par des razzieurs bornouans, soit pour leur permettre de s'engager comme auxiliaire dans l'armée bornouane ${ }^{78}$.

$\boldsymbol{D}_{\text {ans le cas du Sahel central au XIX }}$ siècle, l'idée d'une identité ethnique marquée par des scarifications immuables et figées à travers les générations et même à travers la vie d'un individu ne tient pas dès lors qu'on la confronte aux sources. En effet, les scarifications ne sont pas des marques génériques portées de la même manière par un groupe donné, elles révèlent des pratiques aux fonctions diverses obéissant à des logiques multiples (politique, identitaire, médicale, sociale ou esthétique). Plus précisément, ce n'est pas l'appartenance à une ethnie que l'on porte sur son visage mais plutôt la référence à un lieu ou à un groupe qui n'est la plupart du temps ni ethnique ni tribal mais politique ${ }^{79}$. Il n'est pas nécessaire d'être originaire d'un groupe ou d'y avoir ses ancêtres pour en porter les marques, mais plutôt d'y être accepté, d'en reconnaître les règles et de contribuer à son fonctionnement, par l'impôt ou la participation militaire. Les marques authentifient donc une forme de citoyenneté selon un modèle propre à chaque groupe politique (kasa en haoussa $)^{80}$.

La peau du visage et du corps marquée à différents moments de la vie pour se protéger, se soigner, s'embellir, attirer la chance ou conjurer le mauvais sort peut être lue comme un faisceau de signes ou d'hypersignes et être utilisée selon deux formes de lecture différenciées de l'identité, l'une publique, l'autre intime. Un proverbe haoussa dit : voir est mieux qu'entendre (Ganin ya fi ji $)^{81}$. Ainsi, la chose vue est plus importante que la chose entendue et c'est par la vue que l'on identifie l'autre en se servant du souvenir des modifications corporelles de son corps. Cette mémoire de la peau permet d'attester et de prouver l'identité selon des

\footnotetext{
77. El Hadj Ahmed ben Mohammed el-Fellati est un personnage important du sultanat de Sokoto, il est le gendre de l'émir Muhammad Bello de Kano et son conseiller. Sidney Kanya-Forstner, Paul Lovejoy (eds), Pilgrims, Interpreters and Agents: French Reconnaissance Reports on the Sokoto Caliphate and Borno, 1891-1895, Madison, African Studies Program University of Wisconsin-Madison, 1997, présentation du personnage p. 2-5 et citation p. 39.

78. Information recueillie en 1995 par Christian Seignobos dans l'entourage de Ahmadou Mati, sultan de Guirvidig. Christian Seignobos, Fabien Jamin, La case obus : histoire et reconstitution, Marseille, Éditions Parenthèses, 2004, p. 122.

79. H. Gengenbach souligne, elle aussi, que les scarifications sont liées plutôt à un lieu qu'à une ethnicité. H. Gengenbach, « Boundaries of Beauty: Tattooed Secrets of Women's History in Magude District, Southern Mozambique », art. cité, p. 121. M. Vaughan, elle, a montré comment l'analyse des scarifications permettaient d'observer des catégories ethniques en train de se créer dans le contexte très troublé du tournant du $\mathrm{XX}^{\mathrm{e}}$ siècle au Nyasaland. M. Vaughan, «Scarification in Africa. Re-reading Colonial Evidence », art. cité, p. 395.

80. G. Nicolas, «Les catégories d'ethnie et de fraction ethnique au sein du système social hausa », Cabiers d'études africaines, 15 (59), 1975, p. 408.

81. Proverbe 54, dans Captain G. Merrick, Hausa Proverbs, op. cit., p. 119.
} 
procédures codifiées. Certes, l'identification de l'individu repose sur les relations d'interconnaissance, mais la lecture des signes sur la peau apparaît néanmoins comme l'un des moyens les plus sûrs d'établir l'identité par la preuve ${ }^{82}$.

Camille Lefebvre est historienne, chargée de recherches au CNRS à I'Institut des mondes africains IMAF. Ses travaux portent sur I'histoire du Sahel central (Niger, Nord Nigeria, Sud libyen et algérien) aux XIXe et XXe siècles, notamment sur l'histoire de l'exploration, du territoire et des frontières, ainsi que sur la colonisation. Elle a publié récemment Frontières de sable, frontières de papier. Histoire de territoires et de frontières, du jihad de Sokoto à la colonisation française du Niger, $X I X^{\mathrm{e}}-X X^{\mathrm{e}}$ siècles (Paris, Publications de la Sorbonne, 2015) ; a dirigé, avec Marie-Laure Derat, le dossier « Histoires de territoires. Conception de l'espace et appropriation du territoire en Afrique jusqu'au XIXe siècle ", Afriques. Débats, méthodes et terrains d'histoire (2, automne 2010); publié également « Le temps des lettres. Échanges diplomatiques entre sultans, émirs et officiers français, Niger 1899-1903 ", Monde(s) (5 (1), 2014), p. 57-80); « La décolonisation d'un lieu commun. L'artificialité des frontières africaines : un legs intellectuel colonial devenu étendard de l'anticolonialisme ", Revue d'histoire des sciences humaines (24, 2011, p. 77-104); " We Have Tailored Africa: French Colonialism and the "Artificiality" of Africa's Borders in the Interwar Period ", Journal of Historical Geography (37, 2011, p. 191-202) ; « Itinéraires de sable. Parole, geste et écrit au Soudan central XIXe s. ", Annales HSS (4, 2009, p. 797-824).

camillelefebvre@yahoo.fr

82. Je remercie Roufai Ali, Charlotte Braillon, Djibrilla Koutchi, Richard Kuba, Christian Seignobos et Robin Seignobos pour leur aide et Aïssatou Mbodj-Pouye, Nadège Ragaru et les deux rapporteurs anonymes de Critique internationale pour leurs relectures attentives et stimulantes qui m'ont aidée à améliorer ce texte. 


\section{Critique internationale}

SciencesPo. CNRS $_{\text {CNR }}^{\text {CNIS }}$

\section{Thema}

\section{Voir l'histoire : sources visuelles et écriture du regard}

En sciences sociales, une sollicitation croissante des sources visuelles s'observe depuis quelques années. L'objectif de ce dossier est donc d'interroger la manière dont le visuel - chose vue, acte de voir et régime de visibilité - a transformé l'étude et l'écriture du passé. Au-delà d'une analyse du renouvellement de la documentation, la finalité est d'historiciser la contribution des savoirs de la vision au façonnage d'époques singulières. Le regard que l'on se propose d'étudier est également celui de l'historien, lorsqu'il fait récit de sources visuelles et scripturales. À l'édification d'un gué entre l'hier et l'aujourd'hui, que peut apporter une instrumentation imagée, imaginée parfois aussi, en un temps où les historiens ne cessent d'interroger leur capacité à écrire une histoire qui serait lue?

\section{Varia}

Le concept de clientélisme résiste-t-il à la participation populaire ?

Une comparaison Brésil-Venezuela

par Marie-Hélène Sa Vilas Boas et Federico Tarragoni

\section{Circulations révolutionnaires, légitimations croisées : Cuba-Nicaragua-Venezuela} par Marie-Laure Geoffray et Jan Verlin

Le vieillissement actif : élaboration, légitimation et tentatives de diffusion d'un référentiel transversal en Europe

par Laure Kaeser et Pierre-Alain Roch

\section{État de littérature}

Actualité de la recherche sur le mouvement communiste et la lutte armée aux Philippines

(Gwenola Ricordeau)

\section{Derniers thema parus:}

Communismes et circulations transnationales

\section{Presses de Sciences Po}

117 , boulevard Saint-Germain - 75006 Paris - France

Tél. : +33 (0)1 45498364 - Fax : +33 (0)1 45498334 - Diffusion/distribution CDE/SODIS

www.pressesdesciencespo.fr

Retrouvez la revue sur www.cairn.info et www.persee.fr

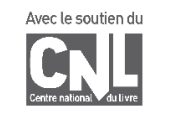

wrww.centrenationaldulivre.fr

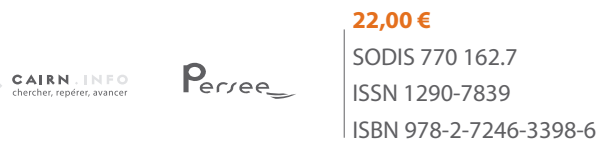

SciencesPo. Iues Presses

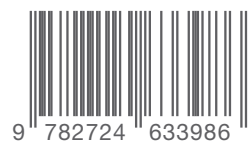

